\title{
A case of new-onset SLE during pregnancy an initial mani- festation with all diagnostic criteria: The challenges of diagnosis and managing a complex disease in a low resource setting
}

\author{
S Kamsanª, B Subhanib, M Rishardc, J Akarawita ${ }^{d}$
}

\begin{abstract}
Systemic lupus erythematosus (SLE) is a complex chronic multiorgan autoimmune disease. When newly diagnosed during pregnancy or puerperium, it is known as new-onset SLE during pregnancy. Delayed or missed diagnosis of newonset SLE during pregnancy may occur due to similar clinical manifestation of SLE with normal physiological changes of pregnancy and lupus nephritis with preeclampsia. New-onset SLE during pregnancy has major organ involvement with poor maternal and foetal outcomes. Management options are limited due to teratogenic side effects of drugs in pregnancy. This is further compounded in a low resource setting with lack of health seeking behaviours and inadequate access to timely care. Multidisciplinary management with close medical, obstetric, neonatal monitoring, patient education and improved access to care is essential for better outcomes. Our aim is to report a 19 year old primigravida with new onset SLE in pregnancy and multiorgan involvement fulfilling all criteria for diagnosis of SLE at the first presentation.
\end{abstract}

Key words: systemic lupus erythematosus (SLE), anti dsDNA antibody, lupus nephritis, neonatal lupus, low resource setting

\section{Introduction}

SLE is an idiopathic chronic-inflammatory-multiorganautoimmune-connective-tissue-disease affecting multiple-organs in the body ${ }^{1}$. It is a multifactorial disease with evidence of genetic susceptibility, environmental effects, and disturbances in both innate and adaptive immunity ${ }^{2}$. Increased SLE disease activity is expected during pregnancy because of increased levels of oestrogen, prolactin and T-helper cell 2 cytokines ${ }^{1,3}$. Pregnancy is associated with an increased risk of SLE flares maybe misdiagnosed due to overlapping features. There are numerous maternaland-foetal-complications ${ }^{4}$. SLE in pregnancy is a complex-disease which should be managed by a team with proper expertise for both investigation and management ${ }^{5}$.

Sri Lanka Journal of Obstetrics and Gynaecology 2021; 43: 185-189

DOI: http://doi.org/10.4038/sljog.v43i3.7998

${ }^{a}$ Registrar in Obstetrics and Gynaecology, De Soysa Hospital for Women, Colombo, Sri Lanka

${ }^{\mathrm{b}}$ Medical Officer in Neurology, National Hospital of Sri Lanka, Colombo, Sri Lanka

c Senior Lecturer, Department of Obstetrics and Gynaecology, Faculty of Medicine, University of Colombo, Sri Lanka

${ }^{\mathrm{d}}$ Consultant Dermatologist, National Hospital of Sri Lanka, Colombo, Sri Lanka

Correspondence: BS, e-mail: brabaharan197subhani@gmail.com

D https://orcid.org/0000-0003-4865-3791

Received $7^{\text {th }}$ June 2021

Accepted $10^{\text {th }}$ October 2021

This is an open-access article distributed under the terms of the Creative Commons Attribution 4.0 International License, which permits unrestricted use, distribution and reproduction in any medium provided the original author and source are credited. 


\section{Case report}

A 19-year-old primigravida at a period-of-gestation of 22 weeks presented with recurrent erythematous skinlesions for 3 months duration. Rash was noted around the abdomen with pruritus. The patient had attributed the rash to be due to God's illness and sought localherbal treatments and presented following subsequent deterioration. There was arthralgia of the small joints. On examination, she was afebrile, pale, anicteric with a diffuse erythematous initially maculopapular (Figure 1), pustular and vesicobullous rash (Figure 2) which appeared as a toxic epidermal necrotic like rash. Painless-oral-ulcers (Figure 3) and patchy nonscarring alopecia were noted. Circulatory, respiratory and abdominal examinations were otherwise compatible with the period of gestation.

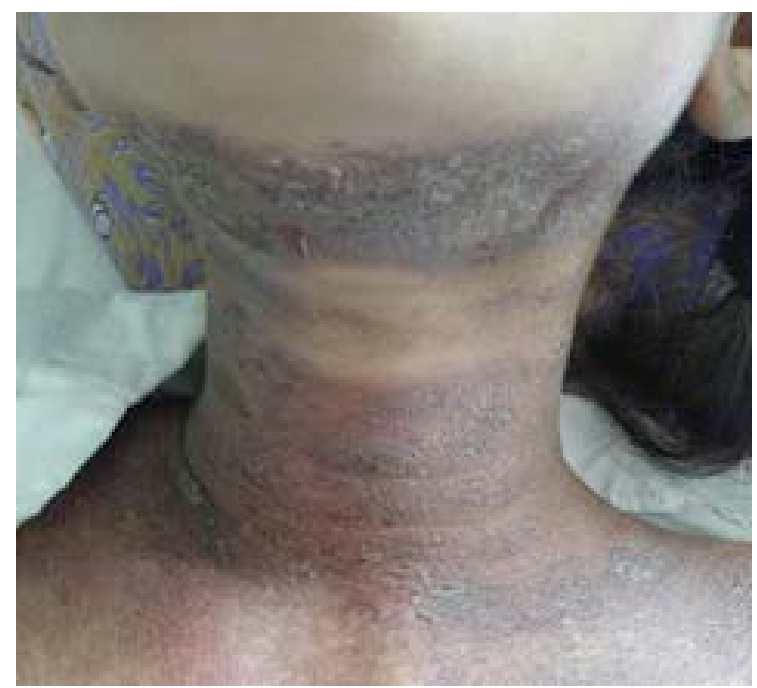

Figure 1. Cutaneous neck rash.

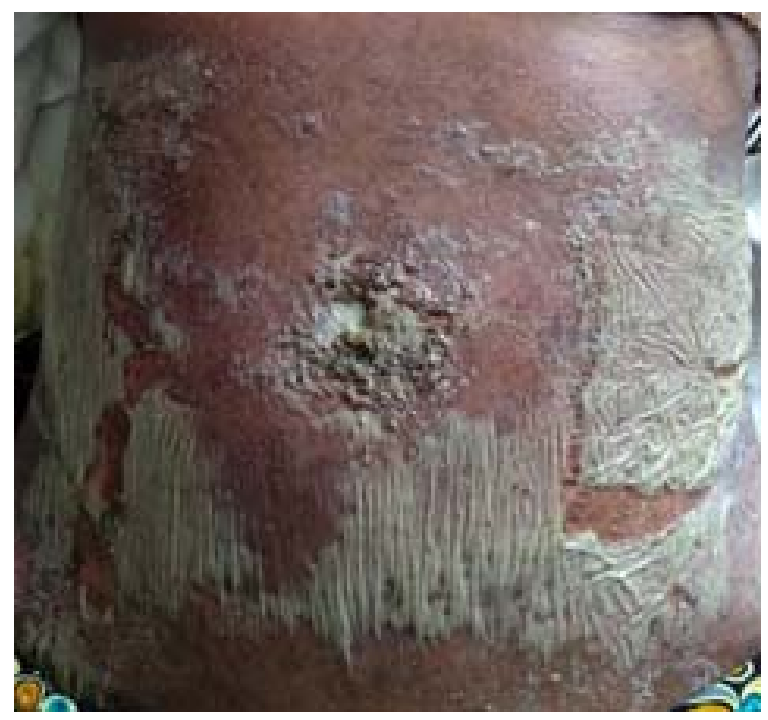

Figure 2. Erythematous pustular rash in the abdomen.

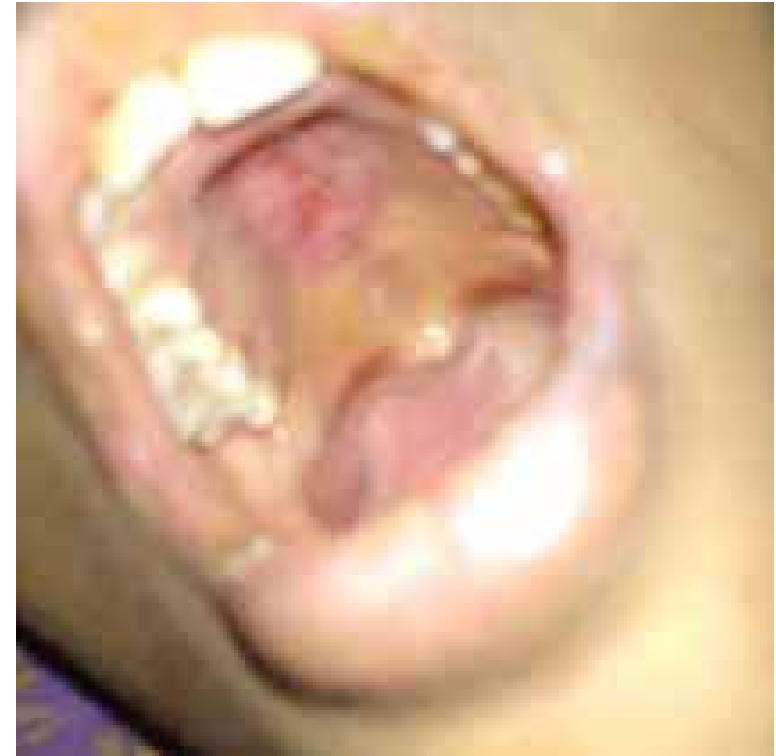

Figure 3. Painless oral ulcer.

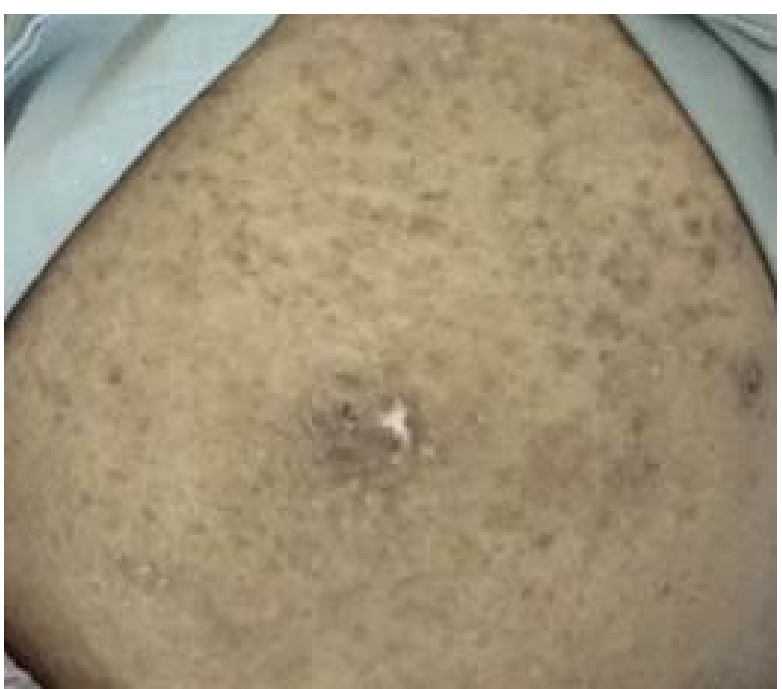

Figure 4. Improvement in the abdominal rash with treatment.

Full blood count and blood picture revealed warmautoimmune-haemolytic-anaemia with a positive DAT (direct antibody test) test and managed with blood transfusions. White cell counts (Neutrophil 79.1\%, Lymphocytes 15.8\%, Eosinophils $1.1 \%$ and platelets (256) were normal. Iron levels were normal. ESR was $50 \mathrm{~mm} / 1 \mathrm{st}$ hour and CRP were $32 \mathrm{mg} / \mathrm{L}$. urine full report (UFR) showed microscopic haematuria and proteinuria with granular casts. uPCR (urine protein creatinine ratio) was 1.16 . Serum creatinine was 0.71 . Urinary protein creatinine ratio was high. Liver profile was normal. Ultrasound abdomen revealed mildly 
increased renal parenchymal echogenicity. Immunological tests revealed a positive-ANA, anti-ds DNAantibodies and low-complement-levels (C3 and C4). Anti Ra, Lo and anti-phospholipid screen were negative. Renal-biopsy revealed class 3 lupus-nephritis. Patient had a systemic-lupus-erythematosus-disease-activityindex (SLEPDAI) score of 25 indicating a severe-flare of SLE. There was an initial suspicion of sepsis due to the blistering lesions, the wound-swab revealed presence of pseudomonas and she was treated with IV antibiotics with pseudomonas-cover. After the infection subsided, she was started on prednisolone $30 \mathrm{mg}$ daily initially, and then reduced to $20 \mathrm{mg}$ daily along with hydroxychloroquine $100 \mathrm{mg}$ twice daily and low dose aspirin $75 \mathrm{mg}$ once daily (Figure 4).

Two-weeks later the patient developed acute-psychosis. MRI-brain and MR-venogram were normal. She was managed as pregnancy-induced-psychosis with olanzapine $5 \mathrm{mg}$ daily. Growth-assessment showed a small-for-gestational-age-foetus with satisfactory umbilical-artery-doppler-parameters and adequate volume of liquor with no identifiable anomalies. Baby was delivered by a caesarean-section at 37 weeks. Neonatal-examination did not show any features of neonatal-lupus. Immediate postpartum-period was unremarkable and patient was discharged with her routine medications to be followed up by the medical team. At each of these stages there were significant delays in primary-access to health-care-services reduced health-seeking behaviour on the part of the patient and her family and subsequent diagnosis and management of the patient as access to advanced immunological investigations is scarce even in the tertiary-sector of the country and there also the oftenpoor acceptance of both the diagnosis and treatment due to low-levels of education and understanding of the implications of the disease.

\section{Discussion}

The original American College of Rheumatologists (ACR) classification required the presence of 4 of 11 criteria which include malar rash, discoid rash, photosensitivity, oral ulcers, arthritis, serositis, renal disorder, neurologic disorder, hematologic disorder, immunologic disorder and antinuclear antibody. The European league against rheumatism (EULAR)/ ACR subsequently revised the criteria in 2019 for SLE which require fulfilment of at least four criteria, with at least one clinical criterion and one immunologic criterion or lupus nephritis as the sole clinical criterion in the presence of ANA or anti-dsDNA antibodies. Clinical criteria include acute cutaneous lupus, chronic cutaneous lupus, oral ulcers-palate, nonscarring alopecia (diffuse thinning or hair fragility with visible broken hairs), synovitis involving two or more joints, characterized by swelling or effusion or tenderness in two or more joints and thirty minutes or more of morning stiffness, serositis, renal, neurologic, haemolytic anaemia, leukopenia ( $<4000 / \mathrm{mm} 3$ at least once) and thrombocytopenia $(<100,000 / \mathrm{mm} 3)$ at least once. Immunological criteria include ANA above laboratory reference range, anti-dsDNA above laboratory reference range, except ELISA: twice above laboratory, anti-Sm, antiphospholipid antibody: any of the following low complement and direct Coombs's test in the absence of haemolytic anaemia ${ }^{6}$.

This new-onset-SLE-during-pregnancy is observed around $0.014 / 1000$ person-years ${ }^{7,8}$. New-onset-SLEduring-pregnancy tends to present with thrombocytopenia-and-renal-involvement early compared with non-pregnant patients but our patient did not have any evidence of thrombocytopenia though there was renal-involvement at diagnosis ${ }^{8,9}$. Toxic-epidermalnecrolysis-(TEN)-like responses have been documented in SLE but is relatively uncommon and there is no documented literature of SLE related TEN like lesions documented during pregnancy $y^{10,11}$. Coexistentinfection and topical-herbal-treatment along with pregnancy may cause a significant change in the presentation of rashes which contributed to delay in diagnosis in our patient ${ }^{12}$. This patient presented with all 11 features of SLE the original diagnostic classification which is rather unusual and rarely documented at the onset though many develop these features over a protracted-period ${ }^{13}$.

Close monitoring of disease-activity during-pregnancy in SLE patients with serological-activity is important as there is a high-risk of complications of the disease as the clinical features may be confounded by the normal clinical features of pregnancy ${ }^{8}$.

Glucocorticoids are most commonly used to treat SLE in pregnancy as induction-and-maintenance therapy. Hydroxychloroquine (an antimalarial drug) and azathioprine is relatively safe compared to other immunosuppressive drugs ${ }^{2}$. Progesterone only implants can be used as effective contraception ${ }^{14}$. Maintenance of same treatment doses should be continued following delivery ${ }^{15,16}$. SLE remains a complex disease which needs to be effectively managed with proper input from rheumatologists, nephrologists and obstetricians. 
Hence there is a need for education of patients with regard to the importance of early presentation so that early investigations can be carried out and proper prompt are referrals carried out ${ }^{17}$.

There is a paucity of research, including fewerresources, reduced-access-to-care, decreased fundingfor-research, and financial-barriers-to-care, providershortage, underdiagnosis, and cultural-differences ${ }^{18}$. The chronic-relapsing and unpredictable-nature-of-thedisease, poor-understanding and acceptance of SLE, compounded by a background of poverty, inadequate social-support structures ${ }^{17}$. However despite of these limitations there have been a few studies which have addressed APLS and other associated disorders with significant improvement of the obstetric-outcomes with low-cost-interventions ${ }^{19}$. Despite the strengths of Sri Lanka's public health sector, poor relationships act as an access-barrier and threat-to-access and affordability $^{20,21}$. In health systems with few resources, putting evidence into practice is proving exceedingly difficult due to patient-related acceptability ${ }^{22-23}$.

\section{Conclusion}

Even though diagnosis of SLE during pregnancy is difficult, better understanding of its clinical manifestations and investigation facilities help in early diagnosis and prompt management. There is need for education and improving the health-care-seekingbehaviour of patients in order to improve diagnosis and management of complex-diseases.

\section{Author declarations}

Conflicts of interest: The authors declare that they have no competing interests.

\section{Funding: None}

\section{Acknowledgments}

We would like to acknowledge the support given by the patient, the family members of the patient for allowing us to use photos of the patient, the help provided throughout the process and all the staff at the De Soysa Hospital for Women for their tireless commitment in the management of our patients.

\section{References}

1. Shakya S, Yang Z. New-onset systemic lupus erythematosus during pregnancy: A challenge in diagnosis. Journal of Reproductive Health and Medicine. 2016; 2(2): 63-6.

2. Lisnevskaia L, Murphy G, Isenberg D. Systemic lupus erythematosus. The Lancet. 2014; 384(9957): 1878-88.

3. Radeka G, Novakov-MikicA, Mitic I. Systemic lupus erythematosus in pregnancy - case report. Medicinski pregled. 2005; 58(5-6): 301-7.

4. Cauldwell M, Nelson-Piercy C. Maternal and fetal complications of systemic lupus erythematosus. The Obstetrician \& Gynaecologist 2012; 14(3): 167-74.

5. Lateef A, Petri M. Managing lupus patients during pregnancy. Best Pract Res Clin Rheumatol. 2013;27(3):435-47.

6. Aringer M, Costenbader K, Daikh D, Brinks R, Mosca M, Ramsey-Goldman R, et al. 2019 European League Against Rheumatism/American College of Rheumatology Classification Criteria for Systemic Lupus Erythematosus. Arthritis \& Rheumatology. 2019; 71(9): 1400-12.

7. Mok CC, Tse SM, Ho LY. Is Pregnancy a Risk Factor for the Onset of Systemic Lupus Erythematosus (SLE) in Women of the Reproductive Age: A Population Based Case-Control Study? Annals of the Rheumatic Diseases. 2015; 74(Suppl 2): 569.

8. Zhao C, Zhao J, Huang Y, Wang Z, Wang H, Zhang $\mathrm{H}$, et al. New-onset systemic lupus erythematosus during pregnancy. Clinical Rheumatology. 2013; 32(6): 815-22.

9. Zhang C, Liang M-y, Xu X, Zhang X-w, Chen S. Clinical features of new-onset systemic lupus erythematosus in pregnant patients. Journal of Obstetrics and Gynaecology Research. 2018; 44(2): 234-40.

10. Monga B, Ghosh S, Jain V. Toxic Epidermal Necrolysis-like Rash of Lupus: A Dermatologist's Dilemma. Indian J Dermatol. 2014; 59(4): 401-2.

11. Romero LS, Bari O, Forbess Smith CJ, Schneider JA, Cohen PR. Toxic epidermal necrolysis-like acute cutaneous lupus erythematosus: report of a case and review of the literature. Dermatology online journal. 2018; 24(5).

12. Gayed M, Stathopoulou E, Rhodes B, Johnston T, Knox E. 6.Rash in Lupus pregnancy. Rheumatology Advances in Practice. 2017;1(suppl_1). 
13. Swaak AJG, van den Brink HG, Smeenk RJT, Manger K, Kalden JR, Tosi S, et al. Systemic lupus erythematosus: clinical features in patients with a disease duration of over 10 years, first evaluation. Rheumatology. 1999; 38(10): 953-8.

14. Knight CL, Nelson-Piercy C. Management of systemic lupus erythematosus during pregnancy: challenges and solutions. Open Access Rheumatol. 2017; 9: 37-53.

15. Andreoli L, Bertsias GK, Agmon-Levin N, Brown $\mathrm{S}$, Cervera R, Costedoat-Chalumeau N, et al. EULAR recommendations for women; health and the management of family planning, assisted reproduction, pregnancy and menopause in patients with systemic lupus erythematosus and/or antiphospholipid syndrome. Annals of the Rheumatic Diseases. 2017; 76(3): 476.

16. Roy J, Das P, Datta A. SLE in Pregnancy. Bangabandhu Sheikh Mujib Medical University Journal. 2010; 3.

17. Dhar JP, Sokol RJ. Lupus and pregnancy: complex yet manageable. Clin Med Res. 2006; 4(4): 310-21.

18. Bansal S, Okoye O, Rajora N. Pregnancy and lupus nephritis in developing countries: A systematic review. Saudi Journal of Kidney Diseases and Transplantation. 2020; 31(1): 10-20.

19. Wijeyaratne CN, Galappaththi SLA, Palipane E, Jayawardane D, Dodampahala SH, Tudawe MN, et al. Pregnancy outcomes of antiphospholipid syndrome: In a low resource South Asian setting. Obstetric Medicine. 2016; 9(2): 83-9.

20. Russell S. Treatment-seeking behaviour in urban Sri Lanka: Trusting the state, trusting private providers. Social Science and Medicine (1982). 2005; 61: 1396-407.

21. Garner P, Kale R, Dickson R, Dans T, Salinas R. Getting research findings into practice: implementing research findings in developing countries. BMJ. 1998; 317(7157): 531-5.

22. Siddiqi K, Newell JN. Putting evidence into practice in low-resource settings. Bulletin of the World Health Organization. 2005; 83(12): 881-968.

23. Anselmi L, Lagarde M, Hanson K. Health service availability and health seeking behaviour in resource poor settings: evidence from Mozambique. Health Econ Rev. 2015; 5(1): 62-. 